\title{
NEW PROCEDURE FOR THE TOTAL SYNTHESIS OF CILOSTAMIDE
}

\author{
Seyed Mohammad Seyedi, Hamid Sadeghian and Zahra Arghiani \\ Department of Chemistry, Faculty of Sciences, Ferdowsi University of Mashhad, Mashhad, I. R. Iran
}

E-mail : smseyedi@yahoo.com

\begin{abstract}
An efficient route to synthesise a wide range of $N, N-\mathrm{R}, \mathrm{R}-4$-(2-oxo-1,2-dihydroquinolin-6-yloxy)butanamide, specially Cilostamide $(R=$ methyl and $R=$ cyclohexyl), one of the most selective inhibitors of phosphodiesterase 3 (PDE3) enzyme, from 5-methoxy-2-nitro benzaldehyde with emphasis on the preparation of the carbostyril (2-quinolinone) ring system is reported.
\end{abstract}

Keywords : Cilostamade, 6-hydroxycarbostyril, p-Anisidine, 6-nitro-3-methoxybenzaldehyde

Introduction

Cilostamide 11 is a highly functionalized compound which shows potent activity of phosphodiestrase3 inhibitory (1-4) lead to cardiotonic effects $(5,6)$, inhibition of platelet aggregation (7-9) and increase in the secretion of Insulin-stimulated glucose (10,11).

The early syntheses of cilostamide was involved the using of 6-hydroxycarbostyril 6 as a substrate (12). The latter compound was synthesized by methoxylation and two step demethylation of 2-chloro-6-methoxyquinoline 3 in six days (13). Compound 3 was obtained from 6-methoxyquinoline (Scheme-1) (14,15). The total yield of this procedure was $20 \%$. The synthesis of compound 6 was also reported by DeRuiter and co-workers (16) starting from oxidation of 6-methoxyquinoline in the presence of hydrogen peroxide in glacial acetic acid to give the $\mathrm{N}$-oxide 2 which was converted to 6 -hydroxycarbostiryl 6 (67\% yield, $36 \mathrm{~h}$ total times) (Scheme-2). Using Beckmann rearrangement to synthesis 6-hydroxycarbostyril 6 from related indanone oxime was not suitable due to low yield $(<5 \%)(17-19)$. It is notable that the early synthesis of 6 -hydroxycarbostyril 6 was reported via decarboxylation of 2-hydroxy-6-methoxyquinoline-4-carboxylic acid $(20,21)$ which was obtained by hydrolysis of crude orizanine (22).

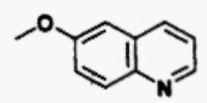

(1)

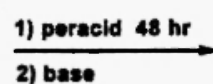

2) base

-
(2)

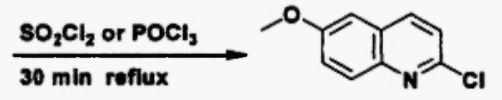

(3)

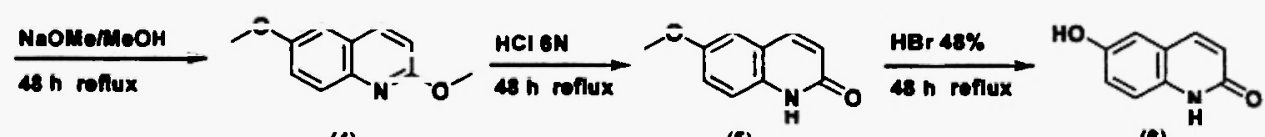

(4)

(5)

Scheme-1

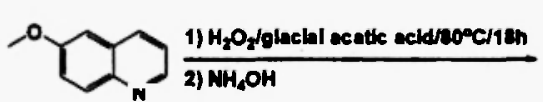

(1)

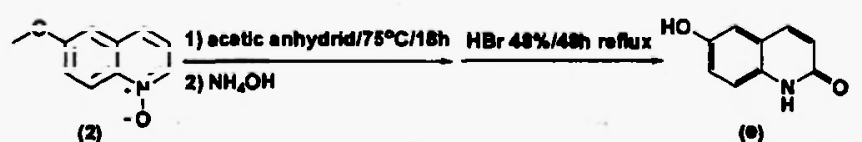

Scheme-2

Converting 5-hydroxy-2-nitrobenzaldehyde 7 to Cilostamide 11 in six step reactions with total yield of $16 \%$ during $64 \mathrm{~h}$ time is also reported (Scheme-3) (4). In this procedure compound 7 was prepared from nitration of protected 3-hydroxy benzaldehyde (23).

Herein we report two procedures for the total synthesis of Cilostamide 11 which are notable from point of view of reaction time and the yield of synthesized Cilostamide 11. In the first procedure the 5-methoxy-2-nitrobenzaldehyde 12 (24) is converted to corresponding nitrocinnamic acid 13 by using malonic acid and piperidine in pyridine (25). Alcoholic solution of $\mathrm{SnCl}_{2} \cdot 2 \mathrm{H}_{2} \mathrm{O}(26)$ reduces compound 13 to 2-amino-5-methoxycinnamic acid 14 in $98 \%$ yield. Acid-catalyzed ring closure of the aminocinnamic acid 14 in dilute hydrochloric acid (27), lead to formation of 6-methoxycarbostyril 5 in $87 \%$ yield. Demethylation of 5 in $48 \%$ aqueous hydrobromic acid produces 6-hydroxycarbostiryl 6 (14). Selective nucleophilic substitution of 6-hydroxy group of 6 by ethyl 4-bromobutyrate in the presence of DBU (1,8-Diazabicyclo[5.4.0]undec-7-ene) afforded Ethyl 4-(1,2-dihydro-2-oxoquinolin-6-yloxy)butanoate 15 in good yield $(13,28,29)$. Hydrolysis of ester 15 in $20 \%$ 
$\mathrm{HCl}$ afforded acid 16. This acid was converted to corresponding acid chloride by using DBU and Isobutyl chloroformate following by direct reaction with $\mathrm{N}$-methylcyclohexylamine to form Cilostamide 11 (Scheme-4).

The second procedure was involved the reaction of $p$-anisidine 17 with cinnamoyl chloride which gave amide 18. FriedelCrafts cyclization of 18 in Chlorobenzene afforded 6-hydroxycarbostiryl 6 (39\% yield) (Scheme-5) (30). Converting the hydroxy compound 6 to Cilostamide 11 was similar to procedure shown in Scheme-4.
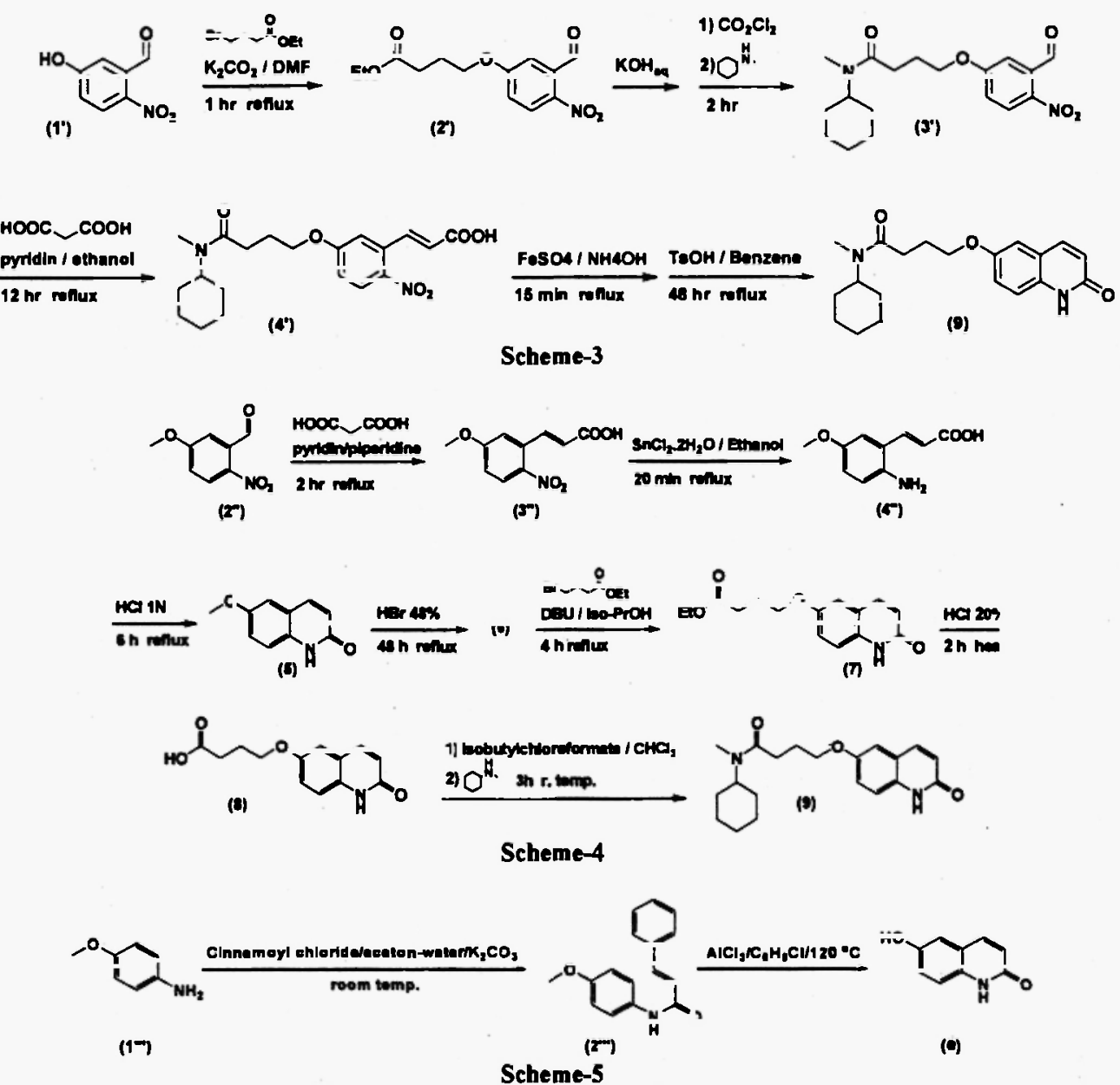

\section{Experimental}

Melting points were recorded on an Electrothermal type 9100 melting point apparatus. The 'HNMR (100 MHz) spectra were recorded on a Bruker AC 100 spectrometer. Chemical shifts are reported in ppm ( $\delta$ ) downfield from tetramethylsilane (TMS). Electron impact (EI) mass spectra were recorded on a Varian Match 7A spectrometer. Elemental analysis was obtained on a Thermo Finnigan Flash EA microanalyzer. All chemicals were purchased from Merck and Fluka Co. and used without further purification.

\section{5-Methoxy-2-nitrocinnamic acid (13)}

$18.1 \mathrm{~g}(0.1 \mathrm{~mol})$ of 6-nitro-3-methoxybenzaldehyde $12(25)$ was added to a mixture of $20.8 \mathrm{~g}(0.2 \mathrm{~mol})$ malonic acid in $40 \mathrm{~mL}$ pyridine. The malonic acid was dissolved by shaking and warming on a steam bath. Piperidine $(1.5 \mathrm{~mL})$ is then added, and the mixture was heated to $80^{\circ} \mathrm{C}$ for $1 \mathrm{~h}$, and then left under reflux for $2 \mathrm{~h}$. After cooling, the reaction mixture was poured into 400 $\mathrm{mL}$ cold water, and while stirring, it was slowly adding $50 \mathrm{~mL}$ of concentrated hydrochloric acid. The resulting was filtered and washed with water. The crude solid acid was dissolved in a solution of $8 \mathrm{~g}$ sodium hydroxide in $300 \mathrm{~mL}$ water. The resulting was filtered and acidified by adding $60 \mathrm{~mL}$ of $\mathrm{HCl}(15 \%)$. The mixture was filtered and the crystalline material was washed with water and dried at $60-70{ }^{\circ} \mathrm{C}$ gave acid $13\left(21.4 \mathrm{~g}, 96 \%, \mathrm{mp} 230^{\circ} \mathrm{C}\right)$. 'HNMR (DMSO-d6): $\delta 3.93(\mathrm{~s}, 3 \mathrm{H}$, OCH3), 6.55 (d, $J=15.6 \mathrm{~Hz}, 1 \mathrm{H},=$ CHCOO), 7.19 (dd, $J=9 \mathrm{~Hz}, 2.6 \mathrm{~Hz}, 1 \mathrm{H}, \mathrm{H}-3), 7.32(\mathrm{~d}, J=2.6 \mathrm{~Hz}, 1 \mathrm{H}, \mathrm{H}-4), 7.97$ (d, $J=$ $15.6 \mathrm{~Hz}, 1 \mathrm{H}, \mathrm{Ph}-\mathrm{CH}=), 8.14(\mathrm{~d}, J=9 \mathrm{~Hz}, 1 \mathrm{H}, \mathrm{H}-6), 12.1-13.3(\mathrm{br}, 1 \mathrm{H},-\mathrm{COOH}) ; \mathrm{MS} \mathrm{m} / \mathrm{z}: 223(\mathrm{M}+), 177(100 \%)$. (Found: C, $53.54 ; \mathrm{H}, 4.10 ; \mathrm{N}, 6.25$. $\mathrm{C}_{11} \mathrm{H}_{11} \mathrm{NO}_{4}$ requires: $\mathrm{C}, 53.82 ; \mathrm{H}, 4.06 ; \mathrm{N}, 6.28 \%$ ) 


\section{5-Methoxy-2-aminocinnamic acid (14)}

A mixture of $20 \mathrm{~g}(0.09 \mathrm{~mol})$ of 5 -methoxy-2-nitrocinnamic acid 13 and $101.5 \mathrm{~g}(0.45 \mathrm{~mol})$ of $\mathrm{SnCl}_{2.2} \mathrm{H}_{2} \mathrm{O}$ in $150 \mathrm{ml}$ of absolute ethanol was refluxed under nitrogen for $20 \mathrm{~min}$. After cooling; the mixture was poured into $500 \mathrm{~mL}$ cold water. The $\mathrm{pH}$ was made $4-5$ by adding sodium bicarbonate. The aminocinnamic acid was extracted by shaking with normal butanol $(3 \times$ $150 \mathrm{~mL}$ ). The separated organic phase treated with charcoal and dried over sodium sulfate. Evaporation of the solvent left 14 $\left(17 \mathrm{~g}, 98 \%, \mathrm{mp} 185^{\circ} \mathrm{C}\right) .{ }^{1} \mathrm{HNMR}$ (DMSO-d6): $\delta 3.66(\mathrm{~s}, 3 \mathrm{H},-\mathrm{OCH}), 6.32(\mathrm{~d}, J=15.6 \mathrm{~Hz}, 1 \mathrm{H},=\mathrm{CHCOO}), 6.71(\mathrm{~m}, 2 \mathrm{H}, \mathrm{H}-3$ \& H-4), 6.98 (d, $J=2.6 \mathrm{~Hz}, 1 \mathrm{H}, \mathrm{H}-6), 7.81(\mathrm{~d}, J=15.6 \mathrm{~Hz}, 1 \mathrm{H}, \mathrm{PhCH}=), 3.3-4.3(\mathrm{br}, 3 \mathrm{H},-\mathrm{COOH} \&-\mathrm{NH} 2) ; \mathrm{MS} m / z: 191$ $(\mathrm{M}+), 78(100 \%)$. (Found: $\mathrm{C}, 68.97 ; \mathrm{H}, 6.92 ; \mathrm{N}, 7.26 . \mathrm{C}_{11} \mathrm{H}_{13} \mathrm{NO}_{2}$ requires: $\mathrm{C}, 69.09 ; \mathrm{H}, 6.85 ; \mathrm{N}, 7.32 \%$ )

\section{6-Methoxycarbostyril (5)}

$15 \mathrm{~g}(0.078 \mathrm{~mol})$ of amino acid 14 was refluxed in $160 \mathrm{~mL} \mathrm{HCl} \mathrm{IN} \mathrm{for} 6 \mathrm{~h}$. After cooling the mixture, pale brown crystals was precipitated which was filtered, washed with water, and dried in oven gave 6-methoxycarbostiryl $5(11.9 \mathrm{~g}, 87 \%$ yield, mp 218 ${ }^{\circ} \mathrm{C}$ ). The small amount of compound 5 recrystallized from acetic acid to get $\mathrm{CHN}$ analysis. 'HNMR (DMSO- $\left.\mathrm{d}_{6}\right): \delta 3.78(\mathrm{~s}, 3 \mathrm{H}$, $\left.-\mathrm{OCH}_{3}\right), 6.47$ (d, $\left.J=9.4 \mathrm{~Hz}, 1 \mathrm{H}, \mathrm{H}-3\right), 7.05-7.32$ (m, 3H, H-5, H-7 \& H-8), 7.83 (d, $\left.J=9.4 \mathrm{~Hz}, 1 \mathrm{H}, \mathrm{H}-4\right), 11.65$ (br, $1 \mathrm{H}$, NHCO); MS m/z: $175(\mathrm{M}+), 175(100 \%)$. (Found: C, 68.77; $\mathrm{H}, 5.24 ; \mathrm{N}, 7.89 . \mathrm{C}_{10} \mathrm{H}_{9} \mathrm{NO}_{2}$ requires: $\mathrm{C}, 68.56 ; \mathrm{H}, 5.18 ; \mathrm{N}$, $8.00 \%)$

\section{6-Hydroxycarbostyril (6)}

$10 \mathrm{~g}(0.058 \mathrm{~mol})$ of compound 5 in $48 \%$ aqueous hydrobromic acid $(200 \mathrm{ml}$.) was boiled for $48 \mathrm{~h}$. Diluting the solution with water $\left(100 \mathrm{~mL}\right.$.) gave white needles of $6\left(8.1 \mathrm{~g}, 88 \%\right.$ yield) which was recrystallized from acetic acid $\left(\mathrm{mp} 334{ }^{\circ} \mathrm{C}\right)$. 1HNMR (DMSO-d6): $\delta 6.44$ (d, $J=9.4 \mathrm{~Hz}, 1 \mathrm{H}, \mathrm{H}-3$ ), $7.01-7.38$ (m, 3H, H-5, H-7 \& H-8), 7.80 (d, $J=9.4 \mathrm{~Hz}, 1 \mathrm{H}, \mathrm{H}-4), 9.03$ (br, $1 \mathrm{H},-$ $\mathrm{OH}), 10.05$ (br, $1 \mathrm{H}, \mathrm{NHCO})$; $\mathrm{MS} m / z: 161(\mathrm{M}+), 83(100 \%)$. (Found: C, 66.97; H, 4.44; N, 8.74. $\mathrm{C}_{10} \mathrm{H}_{9} \mathrm{NO}_{2}$ requires: C, 67.07; $\mathrm{H}, 4.38 ; \mathrm{N}, 8.69 \%)$

\section{Ethyl 4-(1,2-dihydro-2-oxoquinolin-6-yloxy)butanoate (15)}

Ethyl 4-bromobutyrate $(7.3 \mathrm{~g}, 0.033 \mathrm{~mol})$ was added dropwise to a solution of $5 \mathrm{~g}(0.031 \mathrm{~mol})$ of 6-hydroxycarbostyril 6 and 7 $\mathrm{g}$ of DBU in $75 \mathrm{~mL}$ of isopropyl alcohol. The mixture was refluxed for $4 \mathrm{~h}$, the solvent was evaporated off, and the residue was extracted with chloroform. After removal of the solvent, the residue was recrystallized from methanol to give 15 as colorless needles. $\left(7.1 \mathrm{~g}, 83 \%\right.$, mp $\left.153{ }^{\circ} \mathrm{C}\right)$. $1 \mathrm{HNMR}(\mathrm{CDCl})$ ) $\delta 1.31(\mathrm{t}, 3 \mathrm{H}, J=6 \mathrm{~Hz}, \mathrm{CH} 3 \mathrm{CH} 2 \mathrm{OCO}), 2.12(\mathrm{~m}, 2 \mathrm{H},-$ OCH2CH2CH2CO2-), $2.53(\mathrm{t}, 2 \mathrm{H}, J=6 \mathrm{~Hz},-\mathrm{CH} 2 \mathrm{CO} 2-), 4.03(\mathrm{t}, 2 \mathrm{H}, J=6 \mathrm{~Hz},-\mathrm{OCH} 2-), 4.11(\mathrm{q}, 2 \mathrm{H}, J=6 \mathrm{~Hz}$, CH3CH2OCO), 6.69 (d, $1 \mathrm{H}, J=9.4 \mathrm{~Hz}, \mathrm{H}-3), 6.91-7.52(\mathrm{~m}, 3 \mathrm{H}, \mathrm{H}-5, \mathrm{H}-7$ \& H-8), 7.75 (d, $1 \mathrm{H}, J=9.4 \mathrm{~Hz}, \mathrm{H}-4), 12.75$ (br, $1 \mathrm{H}, \mathrm{NHCO})$; $\mathrm{MS} m / z: 275(\mathrm{M}+), 160(100 \%)$. (Found: $\mathrm{C}, 65.37 ; \mathrm{H}, 6.20 ; \mathrm{N}, 5.02 . \mathrm{C}_{15} \mathrm{H}_{17} \mathrm{NO}_{4}$ requires: $\mathrm{C}, 65.44 ; \mathrm{H}, 6.22 ; \mathrm{N}$, $5.09 \%)$

\section{4-(1,2-Dihydro-2-oxoquinolin-6-yloxy)butanoic acid (16)}

A suspention of $15(6 \mathrm{~g}, 0.022 \mathrm{~mol})$ in $60 \mathrm{ml}$ of $20 \% \mathrm{HCl}$ was stirred at $85-90^{\circ} \mathrm{C}$ for $2 \mathrm{~h}$, then cooled. The precipitated crystals were collected, and washed with water. The crystals were recrystallized from DMF-water, oven dried gave $16(5.3 \mathrm{~g}, 99 \%$, mp $265^{\circ} \mathrm{C}$ ). 1HNMR (DMSO-d6): $\delta 1.95(\mathrm{~m}, 2 \mathrm{H},-\mathrm{OCH} 2 \mathrm{CH} 2 \mathrm{CH} 2 \mathrm{CO} 2 \mathrm{H}), 2.39(\mathrm{t}, 2 \mathrm{H}, J=6 \mathrm{~Hz},-\mathrm{CH} 2 \mathrm{CO} 2 \mathrm{H}), 4.01(\mathrm{t}, 2 \mathrm{H}, J=6$ $\mathrm{Hz},-\mathrm{OCH} 2-), 6.46$ (d, $1 \mathrm{H}, J=9.4 \mathrm{~Hz}, \mathrm{H}-3$ ), $7.02-7.35$ (m, 3H, H-5, H-7 \& H-8), 7.79 (d, $J=9.4 \mathrm{~Hz}, 1 \mathrm{H}, \mathrm{H}-4), 11.72$ (br, $1 \mathrm{H}$, $\mathrm{NHCO} \& \mathrm{COOH}$ ); $\mathrm{MS} \mathrm{m} / 2: 247(\mathrm{M}+), 160(100 \%)$. (Found: $\mathrm{C}, 63.31 ; \mathrm{H}, 5.26 ; \mathrm{N}, 5.71 . \mathrm{C}_{13} \mathrm{H}_{13} \mathrm{NO}_{4}$ requires: $\mathrm{C}, 63.15 ; \mathrm{H}$, $5.30 ; \mathrm{N}, 5.67 \%)$

\section{4-(1,2-Dihydro-2-oxoquinolin-6-yloxy)-N-cyclohexyl-N-methylbutanamide (11)}

Isobuthyl chloroformate $(3 \mathrm{~g}, 0.022 \mathrm{~mol})$ was added dropwise to a solution of $4.94 \mathrm{~g}(0.020 \mathrm{~mol})$ of 16 and $3.4 \mathrm{~g}$ of DBU in $100 \mathrm{ml}$ chloroform while stirring in ice-water. After removing the ice-bath, the reaction mixture was stirred at room temperature for $1 \mathrm{~h} .2 .75 \mathrm{~g}(0.024 \mathrm{~mol})$ of $\mathrm{N}$-methylcyclohexylamine was then added dropwise and stirring was continued at room temperature for $3 \mathrm{~h}$. The resulting solution was washed with $0.5 \mathrm{~N} \mathrm{NaOH}(2 \times 50 \mathrm{~mL})$, dil. $\mathrm{HCl}(2 \times 50 \mathrm{~mL})$ and water $(2 \times 50 \mathrm{~mL})$. The non aqueous layer was dried over sodium sulfate. After removing of the solvent under reduced pressure, the residue was recrystalized from $\mathrm{MeOH}-\mathrm{H}_{2} \mathrm{O}$ to give $11\left(5.46 \mathrm{~g}, 80 \%, \mathrm{mp} 187^{\circ} \mathrm{C}\right)$. ${ }^{1} \mathrm{HNMR}\left(\mathrm{CDCl}_{3}\right): \delta$ 0.78-1.97 (m, $10 \mathrm{H}, \mathrm{CH}_{2}$ of cyclohexyl), 1.97-2.74 (m, $\left.4 \mathrm{H},-\mathrm{COCH}_{2} \mathrm{CH}_{2} \mathrm{CH}_{2} \mathrm{O}-\right), 2.82\left(\mathrm{~s}, 3 \mathrm{H}, \mathrm{CH}_{3} \mathrm{NCO}\right), 3.23-3.73(\mathrm{br}, 0.5 \mathrm{H}, \mathrm{CH}$ of cyclohexyl), 4.02 (t, $\left.2 \mathrm{H}, J=6 \mathrm{~Hz},-\mathrm{CH}_{2} \mathrm{O}-\right), 4.17-4.67(\mathrm{br}, 0.5 \mathrm{H}, \mathrm{CH}$ of cyclohexyl), $6.61(\mathrm{~d}, J=9.4 \mathrm{~Hz}, 1 \mathrm{H}, \mathrm{H}-3), 6.78-7.48(\mathrm{~m}, 3 \mathrm{H}, \mathrm{H}-5, \mathrm{H}-7$ \& $\mathrm{H}-8), 7.65(\mathrm{~d}, J=9.4 \mathrm{~Hz}, 1 \mathrm{H}, \mathrm{H}-4), 12.81(\mathrm{br}, 1 \mathrm{H}, \mathrm{NHCO}) ; \mathrm{MS} m / 2: 336(\mathrm{M}+), 176(100 \%)$. (Found: $\mathrm{C}, 71.29 ; \mathrm{H}, 6.03 ; \mathrm{N}$, 8.29. $\mathrm{C}_{20} \mathrm{H}_{20} \mathrm{~N}_{2} \mathrm{O}_{3}$ requires: $\mathrm{C}, 71.41 ; \mathrm{H}, 5.99 ; \mathrm{N}, 8.33 \%$ )

\section{N-(4-methoxyphenyl)cinnamamide (17)}

To a stirred mixture of 4 -methoxyaniline $(9.0 \mathrm{~g}, 73.1 \mathrm{mmol})$, potassium carbonate $(15.0 \mathrm{~g}, 108.5 \mathrm{mmol})$, water $(100 \mathrm{~mL})$, and acetone $(50 \mathrm{~mL})$, was added cinnamoyl chloride $(15.0 \mathrm{~g}, 90.0 \mathrm{mmol})$ at $0{ }^{\circ} \mathrm{C}$ dropwise. The mixture was stirred at $0^{\circ} \mathrm{C}$ for 0.5 $\mathrm{h}$ and then poured into ice-water $(1000 \mathrm{~mL})$. The resulting precipitate was collected by filtration and washed with water. 
Recrystallization from $\mathrm{CHCl}_{3}-\mathrm{MeOH}$ gave 17 as a colorless needles $\left(13.5 \mathrm{~g}, 73 \%\right.$, mp $\left.154-155^{\circ} \mathrm{C}\right){ }^{1}{ }^{\mathrm{H}} \mathrm{NMR}\left(\mathrm{CDCl}_{3}\right) \delta 3.79$ $\left(\mathrm{s}, 3 \mathrm{H},-\mathrm{OCH}_{3}\right), 6.56(\mathrm{~d}, 1 \mathrm{H}, J=15.6 \mathrm{~Hz},=\mathrm{CHCO}), 6.87(\mathrm{~d}, 2 \mathrm{H}, J=9 \mathrm{~Hz}, \mathrm{H}-2$ \& H-6 of methoxyphenyl), 7.28-7.59 (m, $5 \mathrm{H}$, aromatic $\mathrm{H}$ of cinnamamide), 7.54 (d, $2 \mathrm{H}, J=9 \mathrm{~Hz}, \mathrm{H}-3$ \& $\mathrm{H}-5$ of methoxyphenyl), $7.76(\mathrm{~d}, 1 \mathrm{H}, J=15.6 \mathrm{~Hz}=\mathrm{CHPh}$ ); $\mathrm{MS}$ $m / z: 253(\mathrm{M}+), 131(100 \%)$. (Found: $\mathrm{C}, 75.78 ; \mathrm{H}, 6.01 ; \mathrm{N}, 5.49 . \mathrm{C}_{16} \mathrm{H}_{15} \mathrm{NO}_{2}$ requires: $\mathrm{C}, 75.87 ; \mathrm{H}, 5.97 ; \mathrm{N}, 5.53 \%$ )

\section{6-Hydroxycarbostyril (6)}

Aluminum chloride $(13.0 \mathrm{~g}, 97.6 \mathrm{mmol})$ was added portionwise to a suspension of $17(5.0 \mathrm{~g}, 19.8 \mathrm{mmol})$ in chlorobenzene $(100 \mathrm{~mL})$ at $0^{\circ} \mathrm{C}$. The reaction mixture was gradually warmed to $120^{\circ} \mathrm{C}$ and then stirred for $1.5 \mathrm{~h}$. The mixture was poured into ice-water, the resulting sticky precipitate was washed with water and dissolved in ethanol, treated with charcoal and filtered. After removing ethanol the residue was recrystalized from acetic acid to form a white prism crystals $(1.7 \mathrm{~g}, 53 \%$, mp $334{ }^{\circ} \mathrm{C}$ ). (Found: $\mathrm{C}, 67.21 ; \mathrm{H}, 4.47 ; \mathrm{N}, 8.67 . \mathrm{C}_{10} \mathrm{H}_{9} \mathrm{NO}_{2}$ requires: $\mathrm{C}, 67.07 ; \mathrm{H}, 4.38 ; \mathrm{N}, 8.69 \%$ )

\section{Conclusion}

The total yields of the first procedure $(47 \%$, Scheme 4$)$ and of the second procedure $(25 \%$, Scheme 5$)$ are notable when compared with the 20,34 and 16\%, schemes 1,2 and 3 respectively. Although the procedure of scheme 4 , gives higher yield $(47 \%)$ but it takes longer time (67 h for total reactions) in comparison with scheme 5 ( $14 \mathrm{~h}$ for total reactions). The advantage of procedure of scheme 5, in spite of its lower yield, is less synthetic step, shorter time of total reactions and chip regents.

\section{References}

1. M. Endoh, K. Satoh and S. Yamashita, Eur. J. Pharmacol., 66, 43 (1980).

2. C. Lugnier, A. Stierle, A. Beretz, P. Schoeffter, A. Lebec, C. G. Wermuth, J. P. Cazenave and J. C. Stoclet, Biochm. Biophy. Res. Commun., 113, 954 (1983).

3. E. Degerman, P. Belfrage, A. H. Newman, K. C. Rice and V. C. Manganiello, J. Biol. Chem., 262, 5797 (1987).

4. G. H. Jones, M. C. Venuti, R. Alvarez, J. J. Bruno, A. H. Berks and A. Prince., J. Med. Chem., 30, 295 (1987).

5. Y. Inoue, K. Toga, T. Sudo, K. Tachibana, S. Tochizawa, Y. Kimura and Y. Yoshida, H. Hidaka, Br. J. Pharmacol., 130, $231(2000)$.

6. P. G. Phillips, L. Long, M. R. Wilkins and N. W. Morrell, Thorax, 55, A35-A35 (2000).

7. H. Hidaka, H. Hayashi, H. Kohri, H. Kimur, T. Hosokawa, T. Igawa and Y. Saitoh, J. Pharmacol. Exp. Ther., 211, 26 (1979).

8. M. Nishikawa, F. Komada, K. Morita, K. Deguchi and S. Shirakawa, Cellular Signalling, 4, 453 (1992).

9. T. Sudo, K. Tachibana, K. Toga, S. Tochizawa, Y. Inoue, Y. Kimura and H. Hidaka, Biochem. Pharmacol., 59, 347 (2000).

10. P. B. Snyder, Emerging Therapeutic Targets, 3, 4 (1999).

11. M. J. Juan-Fita, M. L. Vargas and J. Hernandez, Eur. J. Pharmacol., 512, 207 (2005).

12. T. Nishi, F. Tabusa, T. Tanaka, H. Ueda, T. Shimizu, T. Kanbe, Y. Kimura and K. Nakagawa, Chem. Pharm. Bull., 3, 852 (1983).

13. R. R. Holmes, J. Conrady, J. Guthrie and R. Mckay, J. Am. Chem. Soc., 76, 2400 (1954).

14. J. Magidson, J. Gen. Chem. (USSR), 7, 1896 (1937).

15. G. B. Bachman and D. E. Cooper, J. Org. Chcm., 9, 302 (1944).

16. J. DeRuiter, A. N. Brubaker, W. L. Whitmer, and J. L. Stein, J. Med. Chem., 29, 2024 (1986).

17. R. E. Gawley, Organic Reaction, 35, 1 (1988).

18. M. J. Tanga, and E. J. Reist, J. Heterocycl. Chem., 23, 747 (1986).

19. Y. Torisawa, T. Nishi and J. I. Minamikawa, Bioorg. Med. Chem., 11, 2205 (2003).

20. J. M. Gulland and R. A. Peters, Biochem. J., 23, 1124 (1929).

21. Y. Sahashi, Biochem. Z., 189, 208 (1927).

22. Y. Sahashi, Sci. Papers. Int. Phys. Chem. Res., 4, 207; 5, 191 (1926).

23. A. Galun, A. Markus and A. Kampf, J. Heterocycl. Chem., 16, 221 (1979).

24. F. A. Mason, J. Chem. Soc., 127, 1195 (1925).

25. Org. Syn. Coll., 4, 327.

26. F. D. Bellamy and K. Ou, Tetrahedron Lett., 25, 839 (1984).

27. W. C. Holzapfel and C. Dwyer, Heterocycles. 48, 215 (1998).

28. T. Fujioka, S. Teramoto, T. Mori, T. Hosokawa, T. Sumida, M. Tominaga, and Y. J. Yabuuchi, J. Med. Chem., 35, 3607 (1992).

29. Z. X. Guo, A. N. Cammidge, A. McKillop and D. C. Horwell, Tetrahedron Lett., 40, 6999 (1999).

30. Manimaran, T.; Thiruvengadam, T. K.; Ramakrishnan, V. T. Synthesis 1975, 739.

Received on September 18, 2007. 